\title{
EXISTENCE OF POSITIVE SOLUTIONS FOR NONLINEAR FRACTIONAL NEUMANN ELLIPTIC EQUATIONS
}

\author{
Haige Ni, Aliang Xia And Xionguun Zheng
}

Abstract. This article is devoted to study the fractional Neumann elliptic problem

$$
\begin{cases}\varepsilon^{2 s}(-\Delta)^{s} u+u=u^{p} & \text { in } \Omega, \\ \partial_{v} u=0 & \text { on } \partial \Omega, \\ u>0 & \text { in } \Omega,\end{cases}
$$

where $\Omega$ is a smooth bounded domain of $\mathbb{R}^{N}, N>2 s, 0<s \leqslant s_{0}<1,1<p<(N+2 s) /(N-$ $2 s), \varepsilon>0$ and $v$ is the outer normal to $\partial \Omega$. We show that there exists at least one nonconstant solution $u_{\varepsilon}$ to this problem provided $\varepsilon$ is small. Moreover, we prove that $u_{\varepsilon} \in L^{\infty}(\Omega)$ by using Moser-Nash iteration.

Mathematics subject classification (2010): 35R11, 35J61, 35A01, 35B45.

Keywords and phrases: Fractional Laplacian, Neumann problem, existence, a priori estimates.

\section{REFERENCES}

[1] C.O. Alves AND O.H. MiYagaKi, Existence and concentration of solution for a class of fractional elliptic equation in $\mathbb{R}^{N}$ via penalization method, Calc. Var. Partial Differential Equations 55 (2016), no. 3, Art. 47, 19 pp.

[2] A. Ambrosetti And P.H. Rabinowitz, Dual variational methods in critical point theory and applications, J. Functional Analysis 14 (1973), 349-381.

[3] V. Ambrosio, Periodic solutions for a pseudo-relativistic Schrödinger equation, Nonlinear Anal. 120 (2015), 262-284.

[4] V. Ambrosio, Periodic solutions for the non-local operator $\left(-\Delta+m^{2}\right)^{s}-m^{2 s}$ with $m \geqslant 0$, Topol. Methods Nonlinear Anal. 49 (2017), no. 1, 75-104.

[5] V. Ambrosio, Multiplicity of positive solutions for a class of fractional Schrödinger equations via penalization method, Ann. Mat. Pura Appl. (4) 196 (2017), no. 6, 2043-2062.

[6] C. Brändle, E. Colorado, A. De Pablo and U. SÁnchez, A concave-convex elliptic problem involving the fractional Laplacian, Proc. Roy. Soc. Edinburgh Sect. A 143 (2013), no. 1, 39-71.

[7] G. CHEN, Singularly perturbed Neumann problem for fractional Schrödinger equations, Sci. China Math., 2017, 60, doi: 10.1007/s11425-016-0420-2.

[8] X. CABrÉAnd J. TAN, Positive solutions of nonlinear problems involving the square root of the Laplacian, Adv. Math. 224 (2010), no. 5, 2052-2093.

[9] L. Caffarelli AND L. Silvestre, An extension problem related to the fractional Laplacian, Comm. Partial Differential Equations 32 (2007) 1245-1260.

[10] L. Caffarelli And P.R. STinga, Fractional elliptic equations, Caccioppoli estimates and regularity, Ann. Inst. H. Poincaré Anal. Non Linéaire 33 (2016), no. 3, 767-807.

[11] S. Dipierro, A. Figalli AND E. VAldinOcI, Strongly nonlocal dislocation dynamics in crys- tals, Comm. Partial Differential Equations 39 (2014), no. 12, 2351-2387.

[12] S. Dipierro, M. Medina, I. Peral and E. Valdinoci, Bifurcation results for a fractional elliptic equation with critical exponent in $\mathbb{R}^{N}$, Manuscripta Math. 153 (2017), no. 1-2, 183-230.

[13] J. DÁvila, M. DEL PINO AND J. WeI, Concentrating standing waves for the fractional nonlinear Schrödinger equation, J. Differential Equations 256 (2014), no. 2, 858-892. 
[14] J. Dávila, M. Del Pino, S. Dipierro and E. Valdinoci, Concentration phenomena for the nonlocal Schrödinger equation with Dirichlet datum, Anal. PDE 8 (2015), no. 5, 1165-1235.

[15] S. Dipierro, X. Ros-OTON AND E. VALdinoci, Nonlocal problems with Neumann boundary conditions, Rev. Mat. Iberoam. 33 (2017), 377-416.

[16] S. Dipierro, N. SoAve AND E. VAldinOci, On stable solutions of boundary reaction-diffusion equations and applications to nonlocal problems with Neumann data, to appear in Indiana Univ. Math. $\mathrm{J}$.

[17] M. M. FAll, F. Mahmoudi AND E. VALDINOCI, Ground states and concentration phenomena for the fractional Schrödinger equation, Nonlinearity 28 (2015), no. 6, 1937-1961.

[18] D. Gilbarg And N.S. Trudinger, Elliptic partial differential equations of second order, Reprint of the 1998 edition. Classics in Mathematics. Springer-Verlag, Berlin, 2001.

[19] C.-S. LIN, W.M. Ni AND I. TAKAGI, Large amplitude stationary solutions to a chemotaxis system, J. Differential Equations 72 (1988), no. 1, 1-27.

[20] R. Musina And A. I. Nazarov, On fractional Laplacians, Comm. Partial Differential Equations 39 (2014), no. 9, 1780-1790.

[21] E. Montefusco, B. Pellacci And G. Verzini, Fractional diffusion with Neumann boundary conditions: the logistic equation, Discrete Contin. Dyn. Syst. Ser. B 18 (2013) 2175-2202.

[22] P.R. Stinga And B. Volzone, Fractional semilinear Neumann problems arising from a fractional Keller-Segel model, Calc. Var. Partial Differential Equations 54 (2015), no. 1, 1009-1042.

[23] P.R. Stinga And J.L. Torrea, Extension problem and Harnack's inequality for some fractional operators, Comm. Partial Differential Equations 35 (2010), no. 11, 2092-2122.

[24] R. SERVADEI AND E. VAldinoci, Variational methods for non-local operators of elliptic type, Discrete Contin. Dyn. Syst. 33 (2013), no. 5, 2105-2137.

[25] R. Servadei And E. VAldinoci, Mountain pass solutions for non-local elliptic operators, J. Math. Anal. Appl. 389 (2012), no. 2, 887-898.

[26] R. SERVADEI AND E. VALDINOCI, Weak and viscosity solutions of the fractional Laplace equation. Publ. Mat. 58 (2014), no. 1, 133-154.

[27] R. Servadei And E. VAldinoci, On the spectrum of two different fractional operators, Proc. Roy. Soc. Edinburgh Sect. A 144 (2014), no. 4, 831-855.

[28] B. Volzone, Symmetrization for fractional Neumann problems, Nonlinear Anal. 147 (2016), 1-25.

[29] M. Willem, Minimax Theorems, Birkhäuser, Boston, 1996.

[30] A. Xia AND J. YANG, Regularity of nonlinear equations for fractional Laplacian, Proc. Amer. Math. Soc. 141 (2013), no. 8, 2665-2672. 